Service social

\title{
Les familles recomposées : pour le meilleur ou pour le pire, une réalité
}

Volume 39, numéro 3, 1990

Familles recomposées après divorce

URI : https://id.erudit.org/iderudit/706497ar

DOI : https://doi.org/10.7202/706497ar

Aller au sommaire du numéro

Éditeur(s)

École de service social de l'Université Laval

\section{ISSN}

1708-1734 (numérique)

Découvrir la revue

Citer ce document

(1990). Les familles recomposées : pour le meilleur ou pour le pire, une réalité. Service social, 39(3), 3-6. https://doi.org/10.7202/706497ar d'utilisation que vous pouvez consulter en ligne.

https://apropos.erudit.org/fr/usagers/politique-dutilisation/ 


\section{AVANT-PROPOS}

\section{Les familles recomposées : pour le meilleur ou pour le pire, une réalité}

Depuis 50 ans la société québécoise a connu des changements radicaux dans ses valeurs, ses normes et ses structures; ces changements se sont répercutés directement sur les structures et le fonctionnement des familles. La hausse fulgurante du nombre de divorces a d'abord amené l'éclatement de la famille dite normale, la famille nucléaire. L'étape suivante fut la famille appelée monoparentale. Et maintenant nous assistons à l'étape qui suit logiquement : la formation de couples et de familles par les adultes qui ont vécu une séparation ou un divorce. Le nombre précis n'est pas connu, à cause des unions de fait non officialisées légalement, mais certains auteurs prévoient que d'ici quelques années, il y aura plus de familles recomposées après divorce que de familles en première union.

Cette situation demande d'être mieux étudiée. Car si le nombre de ces familles est en croissance constante, la perception qu'en a la société, leur image de soi et les services offerts ne connaissent pas un développement aussi rapide. Les modèles appropriés et les normes spécifiques de vie ne sont pas encore précisés. La famille recomposée est encore souvent perçue comme " anormale ", la famille nucléaire primaire demeurant le point de référence positif. Trop souvent, aussitôt qu'un enfant d'une famille recomposée présente des difficultés de comportement ou des problèmes scolaires, la plupart des intervenants sont portés à en attribuer la cause au divorce et à la recomposition - négligeant ainsi d'autres facteurs. Plusieurs auteurs ne mettent en évidence dans les autres formes de familles que les éléments qui font problème de façon dramatique, oubliant qu'après la crise économique des années 30, qui a amené un grand nombre de séparations de fait, et 
après l'industrialisation des années 40-60, qui a provoqué l'éclatement des structures familiales antérieures, la société a dû et a su s'adapter.

Cela s'explique par l'insuffisance des connaissances sur cette nouvelle situation. Les études empiriques sur l'évolution et l'utilisation des ressources de ces familles ne sont pas assez poussées. Les recherches sont peu nombreuses, et les praticiens, dont les connaissances cliniques sont souvent plus approfondies que celles des théoriciens, ne peuvent faire connaître les fruits de leurs analyses. Les intervenants se sentent donc désemparés à cause du manque de connaissances, et souvent, aussi, troublés par des conflits internes venant de leurs valeurs personnelles.

C'est pour offrir des éléments d'analyse et d'action que ce numéro de la revue Service social a été préparé. II propose un éclairage sur ce qui se passe dans les familles recomposées, ce qui est commun aux familles contemporaines et ce qui est particulier à la recomposition. II comprend des résultats de recherche, et aussi des conclusions de praticiens que nous estimons être des praticiens-chercheurs, par I'analyse rigoureuse qu'ils et qu'elles font de leur pratique.

Le contenu est pragmatique : I'existence des familles recomposées pour le meilleur ou pour le pire est une réalité. L'attention exclusive au passé, aux principes et aux normes acceptées mais non suivies concrètement ne sert qu'à augmenter les tensions et à paralyser l'action. Un membre de l'équipe a été fort surpris de constater qu'en France, il existe très peu d'études sur la famille recomposée : les chercheurs ont comme position qu'il s'agit plutôt d'une étape fréquente dans le cycle de la vie familiale. Sans aller aussi loin, nous acceptons le fait de l'existence de ces familles, nous croyons que beaucoup d'entre elles fonctionnent bien, avec des tensions, des transitions, qui peuvent devenir des crises, mais qui sont aussi des sources de développement, et nous proposons de considérer ce qui est vécu dans le quotidien, la signification qu'ont ces faits pour les personnes en cause, de même que les conditions qui peuvent les aider à composer avec la situation et à en reprendre la maîtrise.

Notre perspective est surtout positive et développementale. Nous tenons compte des effets du divorce, du sentiment d'échec et de la souffrance des membres; nous étudions les barrières et les réactions hostiles défensives. Nous accueillons le vécu des familles, avec leurs émotions de tristesse, d'auto-dépréciation par suite de l'échec, de craintes et de colère. Mais nous proposons de ne pas demeurer dans l'étude des premières étapes du deuil, pour nous orienter vers l'étape de réorganisation et de croissance, d'être attentifs aux éléments positifs, aux forces et aux facteurs facilitant I'adaptation active. Nous refusons de demeurer dans le modèle du déficit familial, et nous écou- 
tons le désir d'échanges, de soutien, de partage et de développement chez les membres, qui nous est souvent présenté en profondeur. Contrairement à ceux qui proclament à chaque crise sociale que la famille est menacée de disparaître, nous nous fondons sur la capacité des humains de s'adapter aux conditions de vie et nous postulons que les ressources appropriées existent : il nous faut les connaître, les stimuler, les accompagner.

Notre approche est holistique, c'est-à-dire que nous considérons la situation comme un ensemble, dont les parties sont interdépendantes : le fonctionnement des enfants, des parents, des conjoints et des personnes significatives à l'extérieur de la famille et des environnements sociaux. Nous dépassons la "psychologisation » des comportements, et même leur "familialisation " réductrice. Et nous considérons la dimension éthique, où toutes les actions et les conditions de vie sont étudiées dans leur influence sur le fonctionnement, le développement et la satisfaction des besoins des personnes en cause ainsi que dans le sens qu'elles contribuent à donner à la vie des personnes, à leur modèle du monde et à leurs croyances.

Ce qui amène une analyse politique des situations, centrée sur les relations de pouvoir dans la famille, dans son entourage immédiat, et dans les lieux de décisions politiques et économiques. Qui influence qui, de quelles façons et au bénéfice ou au détriment de qui ? Y a-t-il des groupes vivant une inégalité ou une oppression ? Quelles sont les conditions qui favorisent l'évolution et le développement des familles recomposées, ou qui leur font obstacle ? Quelles sont les sources de soutien social accordé aux différentes formes de famille ? Car il existe des enjeux importants, personnels et sociaux, dans les situations qui nous sont présentées. Et souvent les personnes qui demandent des services, qu'elles soient dans la famille ou dans les organismes sociaux, demandent en même temps de ne pas apporter trop de dérangements...

Un projet aussi ambitieux ne peut être réalisé pleinement dans une revue. II se retrouve néanmoins dans l'ensemble des textes. Un premier article présente les conclusions des principales recherches sur la famille recomposée. Les articles suivants proposent des éléments qui facilitent la relation enfants-beau-parent, et le vécu du nouveau couple conjugal. Un article porte sur une forme particulière d'organisation des relations enfants-parents-beaux-parents : la garde partagée. Puisqu'il n'existe pas de politique sociale sur les familles recomposées, un article présente les grandes lignes de la politique de soutien aux femmes chefs de famille entre la séparation et la recomposition. Enfin, trois articles exposent des modalités d'interventions : I'approche en petits groupes, la plus efficace sur certains points, complétée 
par un document sur la coanimation des groupes; une approche développementale, très appréciée par les familles; et une grille d'évaluation systémique, qui constitue un outil précieux pour organiser les données complexes de ces familles.

Nous espérons que ce contenu, en dépit de son caractère incomplet, sera valable pour faire avancer les connaissances et les services aux familles recomposées et ainsi permettre à plus de familles de connaitre le meilleur, non plus le pire.

L'équipe 